\title{
A FINITENESS THEOREM FOR RICCI CURVATURE IN DIMENSION THREE
}

\author{
SHUN-HUI ZHU
}

A general problem in the study of the relations between curvature and topology lies in understanding which results concerning sectional curvature continue to hold for Ricci curvature. Recently, J. Sha and D. Yang [SY] gave examples which show that certain results such as Gromov's estimate on the Betti numbers and Cheeger-Gromoll's soul theorem, cannot be generalized to the case of Ricci curvature (compare also [AG, An]). In this note, we announce a positive result in this direction concerning finiteness theorems.

The first finiteness results were those of J. Cheeger [Ch] and A. Weinstein [We]. Cheeger's finiteness theorem is the following [Pe]:

Theorem (J. Cheeger, S. Peters). There are only finitely many diffeomorphism types in the class of n-dimensional Riemannian manifolds satisfying

$$
\left|K_{M}\right| \leq \Lambda^{2}, \quad \operatorname{Diam}(M) \leq D, \quad \operatorname{Vol}(M) \geq V,
$$

where $K_{M}$ is the sectional curvature, $\operatorname{Diam}(M)$, the diameter, and $\operatorname{Vol}(M)$, the volume of $M$.

This result is recently generalized in dimensions $\neq 3,4$ by $\mathrm{K}$. Grove and P. Petersen [GP, GPW] to the following:

Theorem (Grove-Petersen-Wu). For $n \neq 3,4$, there are only finitely many diffeomorphism types in the class of n-dimensional Riemannian manifolds satisfying

$$
K_{M} \geq-\Lambda^{2}, \quad \operatorname{Diam}(M) \leq D, \quad \operatorname{Vol}(M) \geq V .
$$

Along this line, it is natural to ask what happens if one replaces sectional curvature by Ricci curvature. The first attempt in answering this question is the following theorem of $\mathbf{M}$. Anderson [An].

Received by the editors July 25, 1989 and, in revised form, November 30, 1989. 1980 Mathematics Subject Classification (1985 Revision). Primary 53C20.

Key words and phrases. Finiteness, Ricci curvature, three manifolds. 
Theorem (M. Anderson). There are only finitely many possibilities of $\pi_{1}$ for the class of n-dimensional Riemannian manifolds satisfying

$$
\operatorname{Ric}_{M} \geq-(n-1) H, \quad \operatorname{Diam}(M) \leq D, \quad \operatorname{Vol}(M) \geq V,
$$

where $\mathrm{Ric}_{M}$ is the Ricci curvature of $M$.

In the same paper [An], M. Anderson gave some examples to show that the crucial point in the proofs of Cheeger's and GrovePetersen-Wu's finiteness theorems, in dimensions $\geq 4$, does not carry over to the case of Ricci curvature. That is, in the class considered in the above result of M. Anderson, there is no lower bound on the length of shortest geodesics. It is still open whether such estimate holds in dimension three.

We prove

Theorem 1. The set of three-dimensional Riemannian manifolds satisfying

$$
\operatorname{Ric}(M) \geq-2 H, \quad \operatorname{Diam}(M) \leq D, \quad \operatorname{Vol}(M) \geq V
$$

contains finitely many homotopy types.

Although there is a close relation between sectional curvature and Ricci curvature in dimension three, our proof does not explore this relation. Instead, the proof is based on the understanding that Ricci curvature is suitable when treating $\pi_{1}$, as illustrated in [An], and hypersurfaces. The proof is a combination of the methods used in [An, SCY, GP] and a volume comparison argument due to J. Cheeger. Some three-dimensional topology is also used.

The basic point is to prove

Proposition. There is a constant $C_{0}=C_{0}(H, D, V)$, such that, for any $r \leq C_{0}, B_{P}(r)$ is contractible in $B_{P}(R \cdot r)$, where $B_{P}(r)$ is the metric ball of radius $r$ centered at $P$.

In contrast to [GP], where a similar statement is shown by exhibiting a deformation, we show this by proving that the inclusion map $I: B_{P}(r) \rightarrow B_{P}(R \cdot r)$ is trivial on $\pi_{1}$ and $\pi_{2}$ for $r$ small. This relies on two different volume comparison arguments.

Denote by $\mathscr{M}(n, H, D, V)$ the set of $n$-dimensional manifolds satisfying $\operatorname{Ric} \geq-(n-1) H$, Diam $\leq D$, Vol $\geq V$. To 
show $I$ is trivial on $\pi_{2}$, we need the following:

Lemma 1. Let $M^{n} \in \mathscr{M}(n, H, D, V)$ and if $K \subset B_{P}(s)$, then there is at most one connected component of $M \backslash K$ which contains a point with distance more than $R(n, H, D, V) \cdot s$ away from $K$.

The argument of showing $I$ is trivial on $\pi_{1}$ follows closely the proof of M. Anderson [An]. But we need in addition a technical lemma from three-dimensional topology.

Lemma 2. Let $M \subset \operatorname{int} N$ be two compact orientable three manifolds with nonempty boundary. Then if $\pi_{2}(M) \rightarrow \pi_{2}(N)$ is trivial, $\pi_{1}(M)$ is torsion free.

Once the proposition is proved, Theorem 1 follows from the center of mass technique employed in [GP, Pet, and Ya].

We also give a noncompact version of Theorem 1 .

Theorem 2. Let $M$ be a complete noncompact three-dimensional Riemannian manifold. If for some point $P$ and a constant $c, M$ satisfies

$$
\begin{aligned}
\operatorname{Ric}_{M} & \geq 0, \\
\operatorname{Vol}\left(B_{P}(r)\right) & \geq c r^{3},
\end{aligned}
$$

then $M$ is contractible.

This gives an affirmative answer to a question of M. Anderson [An] in dimension three. It should also be compared with results of U. Abresch and D. Gromoll [AG], also Z. Shen [Sh].

The author would like to thank professors J. Cheeger, M. Anderson, and R. Skora for their help and encouragement.

\section{REFERENCES}

[AG] U. Abresch and D. Gromoll, On complete manifolds with nonnegative Ricci curvature, preprint.

[An] M. Anderson, Short geodesics and gravitational instantons, preprint.

[CG] J. Cheeger and D. Gromoll, The splitting theorem for manifold of nonnegative Ricci curvature, J. Differential Geom. 6 (1971), 119-129.

[Ch] J. Cheeger, Finiteness theorems for Riemannian manifolds, Amer. J. Math. 92 (1970), 61-74.

[GP] K. Grove and P. Petersen, Bounding homotopy types by geometry, Ann. of Math. 128 (1988), 195-206.

[GWP] K. Grove, P. Petersen and J. Wu, Controlled topology in geometry, Bull. Amer. Math. Soc. 20 (1989), 181-183.

[He] J. Hempel, 3-manifolds, Ann. of Math. Stud. 86 (1976). 
[Pe] S. Peters, Cheeger's finiteness theorem for diffeomorphism classes of Riemannian manifolds, J. Reine Angew. Math. 394 (1984), 77-82.

[Pet] P. Petersen, $A$ finiteness theorem for metric spaces, preprint.

[SCY] R. Schoen and S. T. Yau, Complete three dimensional manifolds with positive Ricci curvature and scalar curvature, Ann. of Math. Stud. 102 (1982), 209-227.

[Sh] Z. Shen, Finiteness and vanishing theorems for complete open Riemannian manifolds, preprint, 1989.

[SY] J. Sha and D. Yang, Examples of manifolds of positive Ricci curvature, J. Differential Geom. 29 (1989), 95-103.

[We] A. Weinstein, On the homotopy type of positively pinched manifolds, Arch. Math. (Basel) 18 (1967), 523-524.

[Ya] T. Yamaguchi, Homotopy finiteness theorems for certain precompact families of Riemannian manifolds, Proc. Amer. Math. Soc. 102 (1988), 660666.

Mathematics Department, State University of New York at Stony Brook, STONY Brook, NEW York, 11794 